\title{
Intervenção Nutricional melhora qualidade da dieta e reduz glicemia em pacientes com aterosclerose manifesta
}

\section{Nutritional intervention improves diet quality and lowers blood glucose in patients with manifest atherosclerotic disease}

Karine Augusto Silva1, Bernadete Weber ${ }^{2}$, Kellen Cristine Silva ${ }^{3}$, Sônia Lopes Pinto 4

\section{RESUMO}

Estudo com o objetivo de avaliar o impacto de um programa de intervenção nutricional na qualidade da dieta e nos marcadores cardiometabólicos de pacientes com história prévia de aterosclerose. Trata-se de um ensaio clínico randomizado com duração de 36 meses, subestudo de um projeto multicêntrico. $O$ grupo intervenção recebeu a Dieta Cardioprotetora Brasileira (DICABr) e o controle foi submetido a orientações nutricionais convencionais conforme diretrizes vigentes. Os grupos foram acompanhados a cada 6 meses por nutricionistas e coletou-se dados antropométricos, bioquímicos, de consumo alimentar e pressão arterial. A adesão à dieta foi avaliada através do índice de adequação à dieta DICABr e o impacto da intervenção a partir da comparação dos componentes da síndrome metabólica após 36 meses. A amostra foi composta por 50 participantes, idade média $61 \pm 8,26$ anos e $68 \%$ apresentavam síndrome metabólica inicialmente. Após 36 meses houve melhora do índice DICABr no grupo intervenção ( $p$ $=0,024)$ e redução no percentual de participantes com glicemia elevada quando comparado ao grupo controle $(p=0,030)$. Para as análises estatísticas utilizou-se teste T-pareado, Wilcoxon e Qui-quadrado de McNemar conforme distribuição das variáveis. Não houve mudança nos dados antropométricos, perfil lipídico e pressórico após 36 meses em nenhum dos 2 grupos.

Palavras-chave: doenças cardiovasculares; síndrome metabólica; consumo de alimentos; estado nutricional; glicemia.

\section{ABSTRACT}

Study with the objective of evaluating the impact of a nutritional intervention program on the quality of the diet and on the cardiometabolic markers of patients with a previous history of atherosclerosis. This is a randomized clinical trial lasting 36 months, a substudy of a multicenter project. The intervention group received the Brazilian Cardioprotective Diet (DICABr) and the control was submitted to conventional nutritional guidelines according to current guidelines. The groups were monitored every 6 months by nutritionists and anthropometric, biochemical, food consumption and blood pressure data were collected. Adherence to the diet was assessed by calculating the index of adequacy to the $\mathrm{DICABr}$ diet and the impact of the intervention by comparing the components of the metabolic syndrome after 36 months. The sample consisted of 50 participants, mean age $61 \pm 8.26$ years and $68 \%$ had metabolic syndrome initially. After 36 months, there was an improvement in the DICABr index in the intervention group $(p$ $=0.024$ ) and a reduction in the percentage of participants with high blood glucose when compared to the control group $(p=0.030)$. For statistical analysis, we used paired t-test, Wilcoxon and McNemar's Chi-square test according to the distribution of variables. There was no change in anthropometric data, lipid and blood pressure profile after 36 months in any of the 2 groups.

Key words: Cardiovascular diseases; metabolic syndrome; food consuption; nutritional status; blood glucose.

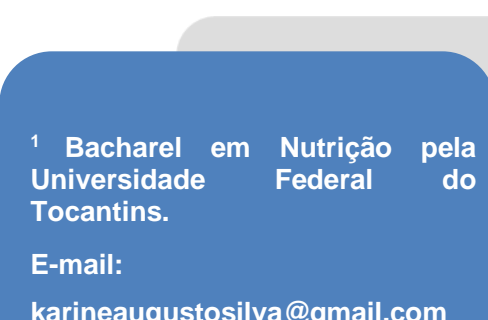

2 Doutora em Ciências da Saúde. Hospital do Coração de São Paulo.

${ }^{3}$ Doutora em Ciência da Nutrição. Universidade Federal do Tocantins.

${ }^{4}$ Doutora em Ciência da Nutrição. Universidade Federal do Tocantins. 


\section{INTRODUÇAO}

As Doenças Cardiovasculares (DCV) são descritas como um grupo de doenças que atingem o coração e os vasos sanguíneos, incluindo assim as doenças coronárias, cerebrovascular, arterial periférica e outras, sendo essas as mais relacionadas à aterosclerose (WHO, 2017).

De acordo com a Organização Mundial de Saúde, em 2016, as DCV eram responsáveis por $31 \%$ de todas as mortes no mundo (17,5 milhões de pessoas), em que 7,4 milhões eram devido à doença coronária e 6,7 milhões a Acidentes Vasculares Cerebrais (AVC) (WHO, 2017). No Brasil o panorama não é diferente, além de serem a maior causa de mortalidade também são responsáveis por gerar os maiores custos com internações hospitalares (IBGE, 2014).

Neste contexto, a alimentação exerce influência não só na prevenção primária às DCV, mas também na prevenção secundária. Recentemente, a Organização das Nações Unidas para a Alimentação e a Agricultura (FAO) publicou um documento que reúne várias evidências que comprovam a associação entre o desenvolvimento de Doenças Crônicas não Transmissíveis (DCNT), entre elas as DCV, e o consumo de alimentos ultraprocessados. Isso ocorre devido às características desse tipo de alimento com conteúdo excessivo de açúcar, gordura saturada, trans e sódio, além do elevado teor calórico e pouca quantidade de fibras e proteínas (MONTEIRO et al., 2019).

Já uma alimentação baseada no consumo de alimentos integrais, ricos em fibras, com preferência para gorduras insaturadas (poli e monoinsaturadas), está associada à redução dos níveis de colesterol total e LDL-c, prevenindo assim um segundo evento aterosclerótico (DE LORGERIL et al., 1999).

Diante disso, este estudo tem como objetivo avaliar o impacto de um programa de intervenção nutricional em pacientes com aterosclerose manifesta, em relação aos parâmetros antropométricos, bioquímicos e de consumo alimentar.

\section{MATERIAIS E METODOS}

Trata-se de um ensaio clínico randomizado que acompanhou pacientes da cidade de Palmas, Tocantins, Brasil, durante um período de 36 meses, comparando dados antropométricos, bioquímicos e de consumo alimentar no baseline e após 36 meses $A$ Universidade Federal do Tocantins - UFT, campus Palmas, foi um dos 35 centros que fizeram parte do estudo nacional multicêntrico coordenado pelo Instituto de Ensino e 
Pesquisa do Hospital do Coração de São Paulo IEP - HCOR, intitulado "Efeito do Programa Alimentar Brasileiro Cardioprotetor na redução de eventos e fatores de risco na prevenção secundária para doença cardiovascular: Um Ensaio Clínico Randomizado (Dica Br)” já publicado (WEBER et al., 2012).

O estudo recebeu aprovação do Comitê de Ética em Pesquisa da Universidade Federal do Tocantins (UFT) no 155/2013, e os participantes assinaram o Termo de Consentimento Livre e Esclarecido para iniciar a coleta de dados.

\section{Amostra}

Fizeram parte do estudo, 50 pacientes de ambos os sexos, seguindo os critérios definidos pelo desenho do estudo (WEBER et al., 2012). Os critérios de inclusão foram: idade $\geq 45$ anos e ter tido algum evento de aterosclerose tais como, doença arterial coronariana, doença cerebrovascular e doença arterial periférica. Foram excluídas pessoas nas seguintes situações: condições psiquiátricas e neurocognitivas que impediam a obtenção de dados fidedignos; expectativa de vida menor que seis meses; período de gravidez ou lactação; insuficiência hepática com história prévia de encefalopatia, insuficiência renal com diálise e insuficiência cardíaca congestiva; transplante de órgãos, gastroplastia, cadeirante e com dificuldades de alimentação via oral. Os participantes do estudo foram divididos em dois grupos: controle e intervenção, através de randomização.

Os indivíduos do grupo controle receberam orientações nutricionais convencionais baseadas nas diretrizes vigentes (SPOSITO et al., 2007; SBC, 2005; SBC, 2010; SBD, 2013-2014).

O grupo intervenção recebeu a dieta cardioprotetora do Programa DICABr, que é baseada nas mesmas recomendações do grupo controle, porém utiliza uma estratégia mnemônica para classificar os grupos de alimentos de acordo com seu risco cardiovascular. Detalhes da elaboração da dieta DICABr foram descritas em publicação anterior (BRASIL, 2018). Todos os participantes do estudo, tanto grupo controle quanto intervenção, passaram por consultas com nutricionistas capacitados pelo projeto, semestralmente, até completar os 36 meses. Adicionalmente, os pacientes do grupo intervenção participaram de 3 atividades coletivas de educação alimentar e nutricional., que utilizavam de estratégias lúdicas para explicar o padrão alimentar cardioprotetor, além de incentivar o consumo de alimentos mais saudáveis através da oferta de preparações feitas baseadas na dieta cardioprotetora para que os participantes pudessem experimentar. Essas ações tinham duração média de uma hora e ocorreram com intervalos de 4 meses. 


\section{Consumo Alimentar e Adesão à Dieta}

As informações referentes ao consumo alimentar foram coletadas pelo método de inquérito Recordatório de 24 horas (R24h) durante as consultas. A partir deste R24h, foi avaliada a adesão dos pacientes de ambos os grupos à dieta cardioprotetora após 36m de acompanhamento. Esta foi avaliada através de um índice dietético, o índice Dica $\mathrm{Br}$, desenvolvido e validado, baseado no Programa Alimentar Cardioprotetor Brasileiro DICABr (TEREZA et al., 2018). Utilizou-se o software Stata® versão 13.0 para as análises estatísticas referentes ao consumo.

\section{Exames Bioquímicos}

A coleta de sangue foi realizada na UFT, por um laboratório de análises clínicas especializado. Os exames solicitados foram: lipidograma (triglicérides, colesterol total, colesterol HDL e colesterol LDL) e glicemia em jejum. Os níveis de normalidade foram avaliados de acordo com a International Diabetes Federation (Glicemia) e SBC (demais exames) (ALBERTI; ZIMMET; SHAW, 2006; SBC, 2017).

\section{Pressão Arterial}

Foi aferida por profissional habilitado, usando um aparelho portátil e digital da marca Orom® sempre no braço esquerdo. A pressão arterial foi considerada normal quando estava menor que 130/85mmHg (ALBERTI; ZIMMET; SHAW, 2006).

\section{Antropometria}

Para a medida do peso foi utilizada balança eletrônica da marca Welmy®. A pesagem foi feita de acordo com as técnicas de Jellife (JELLIFE, 1968). Realizaram-se duas medidas e obteve-se a média entre elas.

Para a medida da altura, foi utilizado um estadiômetro de parede, com precisão de $0,50 \mathrm{~cm}$. Realizaram-se duas medidas e, caso a diferença entre as duas fosse maior que 1 $\mathrm{cm}$ era realizada uma terceira medida.

Utilizando-se os valores de peso em $\mathrm{kg}$ e a altura em metros, o Índice de Massa Corporal (IMC) foi calculado. A classificação do estado nutricional foi realizada de acordo com a preconização da OPAS (2002) e da WHO (1995) (OPAS, 2002; WHO, 1995).

A medida do perímetro da cintura (PC) foi realizada com fita métrica inelástica de 2 metros com precisão de $0,1 \mathrm{~cm}$. A medida foi feita no ponto médio entre a borda inferior do arco costal e a crista ilíaca na linha axilar média conforme Callaway (CALLAWAY, et al, 1988). 


\section{Síndrome Metabólica}

A presença de Síndrome Metabólica (SM) foi determinada conforme o Consenso Mundial para Definição de Síndrome Metabólica da International Diabetes Federation (ALBERTI; ZIMMET; SHAW, 2006).

\section{Análise Estatística}

A análise dos dados foi feita através do software SPSS versão $20.0{ }^{\circledR}$. As variáveis contínuas foram expressas em médias e desvio padrão e as categóricas, em frequência absoluta e relativa. A normalidade dos dados foi testada através do teste de KolmogorovSmnorv e pela avaliação do gráfico de histograma. Quando tinham distribuição paramétrica, aplicou-se o teste T-pareado. Para as variáveis com distribuição não-paramétrica, foi realizado o teste de Wilcoxon. O teste de qui-quadrado de McNemar foi utilizado para testar a associação entre as variáveis categóricas. Para todos os testes estatísticos foi adotada significância menor que $5 \%$.

\section{RESULTADOS}

Fizeram parte do estudo 50 indivíduos, sendo $82 \%$ homens, com média de idade de $61 \pm 8,26$. Cerca de $60 \%$ dos participantes apresentavam Doença Arterial Coronariana (DAC) (TABELA 1).

Tabela 1 - Características demográficas, metabólicas e estado nutricional de pacientes com doença aterosclerótica manifesta, de acordo com grupo de tratamento, no baseline $(n=50)$.

\begin{tabular}{|c|c|c|c|c|c|c|}
\hline \multirow{2}{*}{$\begin{array}{l}\text { Variáveis } \\
\text { Sexo }\end{array}$} & \multicolumn{2}{|c|}{$\begin{array}{l}\text { Controle } \\
(n=24)\end{array}$} & \multicolumn{2}{|c|}{ Intervenção ( $n=26)$} & \multicolumn{2}{|c|}{$\begin{array}{l}\text { Total } \\
(n=50)\end{array}$} \\
\hline & $\mathbf{n}$ & $\%$ & $\mathbf{N}$ & $\%$ & $\mathbf{n}$ & $\%$ \\
\hline Feminino & 8 & 33,3 & 1 & 3,8 & 9 & 18 \\
\hline Masculino & 16 & 66,7 & 25 & 96,2 & 41 & 82 \\
\hline \multicolumn{7}{|l|}{ Grupo Etário } \\
\hline Adultos & 11 & 45,8 & 13 & 50 & 24 & 48 \\
\hline Idosos* $^{*}$ & 13 & 54,2 & 13 & 50 & 26 & 52 \\
\hline \multicolumn{7}{|c|}{ Estado Nutricional } \\
\hline Baixo Peso & 2 & 8,3 & 3 & 11,5 & 5 & 10 \\
\hline Eutrofia & 8 & 33,3 & 7 & 26,9 & 15 & 30 \\
\hline
\end{tabular}


DOI: 10.18605/2175-7275/cereus.v13n2p17-28
SILVA.K, A; WEBER.B; SILVA. K.C; PINTO.S, L.

Intervenção Nutricional melhora qualidade da dieta e reduz glicemia em pacientes com aterosclerose manifesta

\begin{tabular}{lllllll}
\hline Sobrepeso & 6 & 25,0 & 10 & 38,5 & 16 & 32 \\
Obesidade & 8 & 33,3 & 6 & 23,1 & 14 & 28
\end{tabular}

\section{Presença de}

$\begin{array}{lllllll}\text { Síndrome Metabólica } & 18 & 75 & 16 & 61,5 & 34 & 68\end{array}$

Perímetro da Cintura

$\begin{array}{lllllll}\text { Aumentado** } & 21 & 87,5 & 19 & 73,1 & 40 & 80 \\ \text { HDL-c Baixo } & 10 & 41,7 & 11 & 42,3 & 21 & 42\end{array}$

Triglicerídeos

$\begin{array}{lcccccc}\text { Aumentados } & 20 & 83,3 & 19 & 73,1 & 39 & 78 \\ \text { Glicemia Alterada } & 13 & 54,2 & 16 & 61,5 & 29 & 58\end{array}$

Pressão Arterial

$\begin{array}{lllllll}\text { Aumentada } & 23 & 98,4 & 25 & 96,2 & 48 & 96\end{array}$

Fonte: Elaborado pelo autor.

*Acima de 60 anos; **Acima de $80 \mathrm{~cm}$ para mulheres e acima de $92 \mathrm{~cm}$ para homens.

Ao compararmos as médias das variáveis antropométricas, de perfil lipídico, glicêmico e pressórico nas condições basais e após 36 meses não observamos diferenças em ambos os grupos avaliados (Tabela 2).

Tabela 2 - Perfil antropométrico e bioquímico no baseline e após 36 meses de acompanhamento de pacientes com doença aterosclerótica manifesta, de acordo com o grupo de tratamento.

\begin{tabular}{|c|c|c|c|c|c|c|}
\hline \multirow[b]{2}{*}{ Variáveis } & \multicolumn{3}{|c|}{ Intervenção } & \multicolumn{3}{|c|}{ Controle } \\
\hline & $\begin{array}{l}\text { Baseline } \\
\text { (média } \pm \text { ) }\end{array}$ & $\begin{array}{l}36 \text { meses } \\
\text { (média } \pm \text { ) }\end{array}$ & p & $\begin{array}{l}\text { Baseline } \\
\text { (média } \pm \text { ) }\end{array}$ & $\begin{array}{l}36 \text { meses } \\
\text { (média } \pm \text { ) }\end{array}$ & $\mathbf{p}$ \\
\hline Peso(kg) & $76,6 \pm 16,3$ & $79,9 \pm 18,3$ & 0,816 & $71,6 \pm 12,4$ & $72,8 \pm 11,4$ & 0,171 \\
\hline Altura(m) & $1,66 \pm 0,09$ & - & - & $1,60 \pm 0,08$ & - & - \\
\hline $\begin{array}{l}\text { IMC } \\
\left(\mathrm{kg} / \mathrm{m}^{2}\right)\end{array}$ & $27,8 \pm 4,84$ & $28,5 \pm 5,6$ & 0,865 & $27,5 \pm 3,7$ & $27,8 \pm 2,9$ & 0,215 \\
\hline $\mathrm{PC}(\mathrm{cm})$ & $100 \pm 13,5$ & $101,1 \pm 16,5$ & 0,352 & $96,0 \pm 8,9$ & $99,6 \pm 7,0$ & 0,315 \\
\hline CT (mg/dl) & $156,8 \pm 33,1$ & $160,3 \pm 62,6$ & 0,709 & $173,7 \pm 39,7$ & & 0,383 \\
\hline
\end{tabular}


$181,6 \pm 72,8$

$\begin{array}{lcccccc}\text { LDL-c } & 76,7 \pm 37,5 & 88,4 \pm 56,5 & 0,422 & 93,7 \pm 36,8 & 101,8 \pm 56,7 & 0,326 \\ \begin{array}{l}\text { (mg/dl) } \\ \text { HDL-c }\end{array} & & & & & \\ \text { (mg/dl) } & 42,3 \pm 10,2 & 43,1 \pm 11,1 & 0,723 & 46,3 \pm 12,6 & 42,9 \pm 9,3 & 0,720 \\ \text { TG (mg/dl) } & 168,5 \pm 80,6 & 146 \pm 62,9 & 0,103 & 151,5 \pm 73,9 & 195,4 \pm 177,6 & 0,796 \\ \text { Glicemia } & & & & & & \\ \text { (mg/dl) } & 109,1 \pm 30,3 & 99,5 \pm 21,8 & 0,438 & 100,9 \pm 16,2 & 94,7 \pm 15,5 & 0,796\end{array}$

Fonte: Elaborado pelo autor.

IMC: Índice de Massa Corporal; PC - perímetro da cintura; CT - colesterol total; TG - triglicerídeos. Foi utilizado teste t pareado para as variáveis peso, CT, LDL-c, LDL-c e HDL-c; teste de Wilcoxon para as variáveis IMC, PC, TG e Glicemia.

Em relação à SM e seus componentes (perímetro da cintura, pressão arterial, HDL-C, triglicerídeos e glicemia) foi possível observar que após 36 meses não houve mudança do percentual de participantes com SM entre os grupos, no entanto encontramos menor frequência de pacientes com glicemia elevada no grupo intervenção, com p=0,030 (Gráfico $1)$.

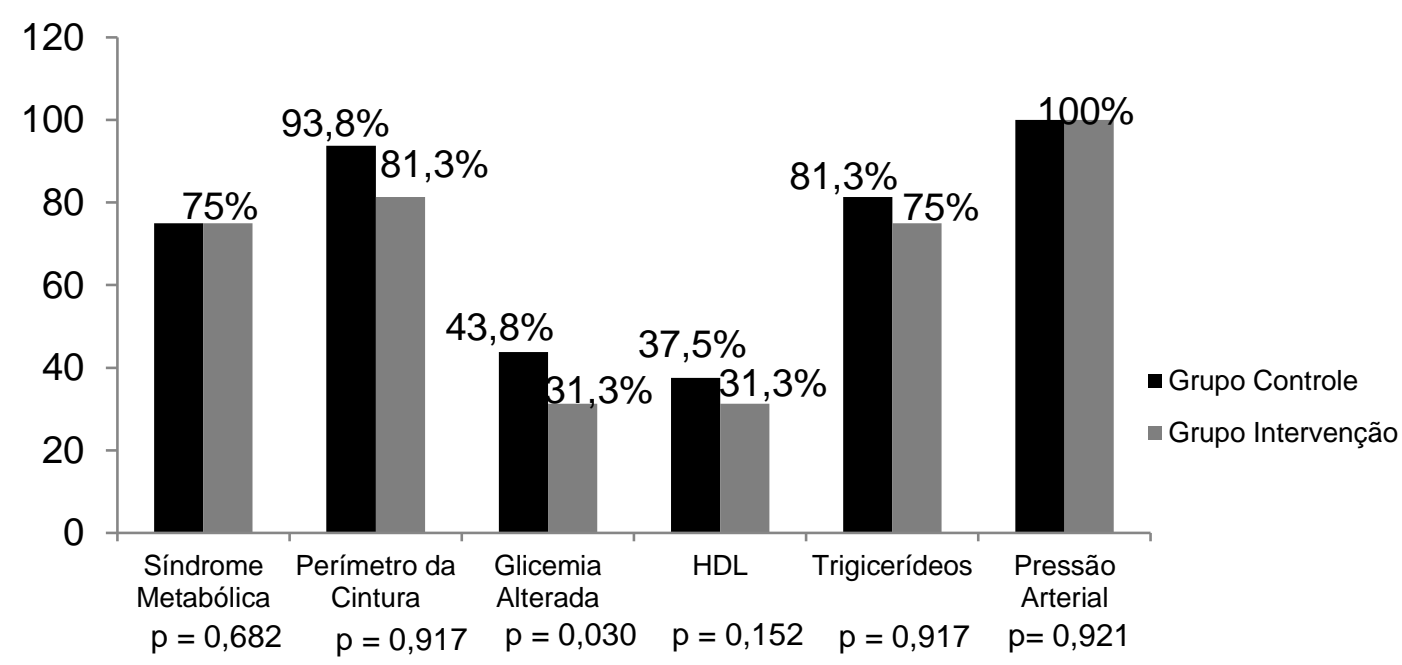

Gráfico 1 - Frequência de Síndrome Metabólica e das alterações dos seus componentes nos pacientes com aterosclerose manifesta do grupo controle e intervenção com $36 \mathrm{~m}$ de acompanhamento $(n=32)$. 
Ao analisar a adesão dos pacientes à dieta cardioprotetora (DICABr), foi possível identificar aumento de 4,4 pontos na média do escore do índice dietético no grupo intervenção, ou seja, o consumo dos grupos alimentares (verde, amarelo, azul e vermelho) aproximou-se das quantidades recomendadas após os 36 meses de acompanhamento em relação as condições basais (Tabela 3). No gráfico 2 identificamos que o percentual de pacientes que melhoraram a pontuação do índice Dica Br após 36 meses foi maior entre os pacientes do grupo intervenção quando comparados ao grupo controle.

Tabela 3 - Escore do índice dietético DICA Br nos pacientes com aterosclerose manifesta no baseline e após 36 meses de acompanhamento, conforme grupo de tratamento $(n=50)$.

\section{Escore do Índice dietético DICA Br}

\begin{tabular}{llll}
\cline { 2 - 3 } Grupos & $\begin{array}{c}\text { Baseline } \\
\text { (média } \pm \text { ) }\end{array}$ & $\begin{array}{c}\text { 36 meses } \\
\text { (média } \pm \text { ) }\end{array}$ & p \\
\hline Intervenção* & $18,3 \pm 7,0$ & $22,7 \pm 7,2$ & 0,024 \\
Controle** $^{*}$ & $20,5 \pm 8,1$ & $17,4 \pm 8,1$ & 0,141
\end{tabular}

Fonte: Elaborado pelo autor.

${ }^{*}$ Condições Basais $n=26,36$ meses $n=16 ;{ }^{* *}$ Condições Basais $n=24,36$ meses $n=16$.

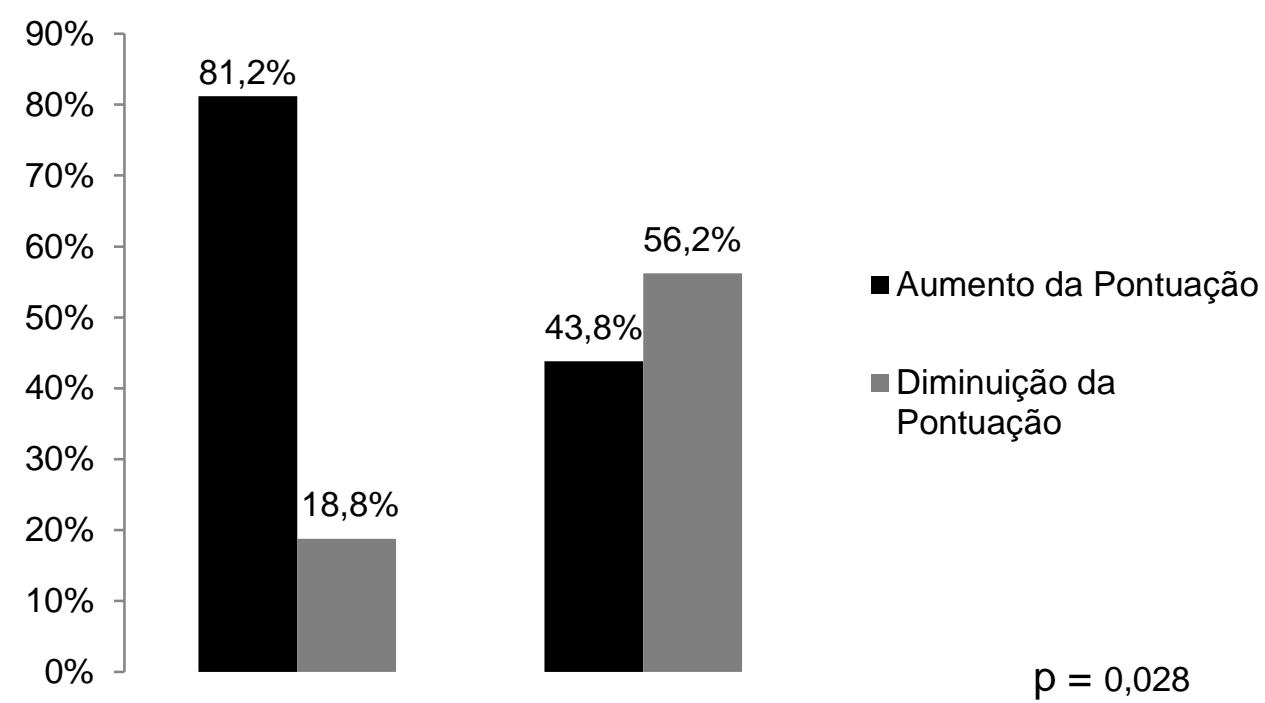

Gráfico 2 - Impacto da intervenção nutricional no índice dietético DICA Br nos pacientes com aterosclerose manifesta após 36 meses de acompanhamento.

\section{DISCUSSÃO}

O índice dietético utilizado para avaliar a adesão dos participantes à dieta cardioprotetora foi validado para os grupos alimentares do DICABr e é capaz de identificar 
maior ou menor grau de adesão, considerando que quanto mais próxima a 40 esteja à pontuação, maior o seguimento das recomendações de porções, o que pode reduzir as chances do indivíduo ser acometido por outro problema de ordem cardiovascular. O índice não estabelece ponto de corte para indicar adequação ou não a dieta, mas sim considera valores mais próximos a 40 (pontuação máxima do índice) como maior grau de adesão e quanto mais distante desse valor, menor grau de adesão (TEREZA et al., 2018). De acordo com os resultados encontrados em nosso estudo, podemos identificar que os participantes do grupo intervenção estavam mais inclinados a seguir a proposta da dieta DicaBr, devido ao aumento no score do índice dietético após 36 meses.

No estudo principal, com mais de 2.000 pacientes, que reuniu dados de todo Brasil, também foi encontrada melhora significativa no consumo alimentar de legumes e frutas no grupo intervenção quando comparado ao controle, após os 36 meses de acompanhamento. Foi constatada também redução significativa na ingestão de calorias, gorduras e colesterol e um aumento no consumo de carboidratos no grupo intervenção em relação ao grupo controle, porém, não houve mudanças nos fatores de risco cardiometabólicos (WEBER et al., 2019).

Mesmo com resultados positivos em relação à melhora na ingestão alimentar dos participantes, ainda estamos longe do ideal. O estudo de Santos et al. (2012) sugere que há baixa adesão a mudanças relacionadas ao estilo de vida, principalmente em relação à alimentação e atividade física. Na mesma ótica está o aporte teórico de Guimarães et al. (2010) baseado na avaliação do grau de conhecimento e adesão a mudanças comportamentais em pacientes com hipertensão e doenças cardiovasculares. A maioria dos pacientes acreditava que a redução no consumo de sal, gordura e açúcar, assim como a prática de atividade física e controle do estresse, traziam benefícios para a saúde; porém, uma minoria tinha adesão a tais recomendações, justificando que as modificações alimentares alteram o prazer em comer e falta de tempo para prática de atividade física e descanso.

A dieta cardioprotetora é baseada nos princípios nutricionais da Dieta Mediterrânea, porém, leva em consideração os costumes alimentares de cada região do Brasil como estratégia para facilitar a adesão dos pacientes a um estilo de vida mais saudável. A Dieta Mediterrânea tem como principais características o elevado consumo de frutas frescas, vegetais, cereais integrais e leguminosas, além de moderada ingestão de peixes, aves, leites e derivados, tendo as nozes e o azeite de oliva como as principais fontes de gordura. 
O consumo de carnes vermelhas e outros alimentos com grande quantidade de gordura saturada não é encorajado (CHANGES, 2010).

Devido à sua composição, a Dieta Mediterrânea está relacionada à prevenção de doenças cardiovasculares, tanto em nível primário quanto secundário, e está associada à redução de alguns componentes da SM. Apresenta elevado teor de ácidos graxos monoinsaturados e menor quantidade de ácidos graxos saturados, estando associada à redução de marcadores inflamatórios e dislipidemia (STEEMBURGO et al., 2007). A presença marcante das fibras também exerce efeitos importantes, sobretudo no que se refere ao metabolismo da glicose e colesterol.

Observamos uma redução do percentual de pacientes com glicemia alterada no grupo intervenção quando comparado ao grupo controle. Uma das possíveis justificativas seria o maior consumo de fibras na dieta, pois seu consumo é estimulado no Programa DICABr. Um estudo com pacientes diabéticos mostrou impacto positivo no controle glicêmico em uma intervenção nutricional, baseada no aumento do consumo de fibras (CARVALHO et al., 2012). As fibras solúveis provocam retardo no esvaziamento gástrico e absorção mais lenta dos carboidratos. Isso retarda a difusão de glicose para a corrente sanguínea, reduzindo o incremento da glicemia e também da secreção de insulina (DALL'ALBA; AZEVEDO, 2010). Além disso, de acordo com a Diretriz Brasileira de Diabetes, dietas ricas em ácidos graxos monoinsaturados auxiliam no controle glicêmico e reduz marcadores de risco cardiovascular. Sendo assim, o perfil da dieta cardioprotetora pode ter contribuído para a redução da glicemia dos participantes do grupo intervenção (SBD, 2017-2018).

Tivemos uma perda considerável da amostra inicial ao longo do estudo, sendo $36 \%$ da amostra total (33,3\% do controle e 38,4\% intervenção). Isso pode interferir na não detecção de alterações antropométricas e no perfil lipídico e pressórico ao fim do estudo, mesmo com a melhora encontrada no índice dietético. Outra limitação é que a amostra do estudo não é representativa da população de Palmas, pois não foi feito cálculo amostral, já que a cidade era apenas um dos centros dentre outros espalhados pelo Brasil que faziam parte do Estudo em âmbito Nacional.

O estudo apresenta metodologia bem delineada, com critérios rigorosos de inclusão e exclusão. Os pacientes foram randomizados, o que exclui vieses de seleção no momento da divisão dos grupos, além de ser o primeiro estudo clínico randomizado que avalia o impacto de uma intervenção nutricional, em pacientes com doença aterosclerótica manifesta, da região Norte do Brasil. 


\section{CONSIDERAÇOES FINAIS}

Neste estudo, observamos que a intervenção nutricional teve impacto positivo em relação à adesão dos pacientes a dieta cardioprotetora e, isso, levou a uma redução significativa nos níveis de glicose sérica no grupo intervenção.

ALBERTI, K. G. M. M.; ZIMMET, P.; SHAW, J. Metabolic syndrome - a new world-wide definition. A consensus Statement from the International Diabetes Federation. Diabetic Medicine. [S.I.], v. 23, p. 469-480, abr. 2006.

BRASIL. Alimentação Cardioprotetora: Manual de Orientações para Profissionais de Saúde da Atenção Básica. Brasília: Ministério da Saúde, 2018. Disponível em: <http://189.28.128.100/dab/docs/portaldab/publicacoes/alimentacao_cardioprotetora_orien _pro_saude_ab.pdf>. Acesso em: out. 2018.

CALLAWAY, C. W. et al. Anthropometric Standardizing Reference Manual. Champaign: Human Kinetics Books, 1988.

CARVALHO, F. S. et al. Importância da orientação nutricional e do teor de fibras da dieta no controle glicêmico de pacientes diabéticos tipo 2 sob intervenção educacional intensiva. Arquivos Brasileiros de Endocrinologia \& Metabologia. Arquivos Brasileiros de Endocrinologia e Metabologia. São Paulo: v. 56, n. 2, p. 110-119, 2012.

CHANGES, M. C. D. T. Efeito da dieta tipo Mediterrânea na função endotelial e inflamação na aterosclerose: estudo comparativo com a dieta TLC (" Therapeutic Lifestyle Changes ", do NCEP - ATPIII ). Tese (Doutorado) - Universidade de São Paulo, 2010. Disponível em: <https://www.teses.usp.br/teses/disponiveis/5/5131/tde-24062010143245/pt-br.php>. Acesso em: dez. 2018.

DALL'ALBA, V.; DE AZEVEDO, M. J. Papel das Fibras Alimentares Sobre o Controle Glicêmico, Perfil Lipídico e Pressão Arterial em Pacientes com Diabetes Melito Tipo 2. Rev HCPA. Porto Alegre, v. 30, n. 4, p. 363-371, nov. 2010.

DE LORGERIL, M. et al. Mediterranean diet, traditional risk factors, and the rate of cardiovascular complications after myocardial infarction. Circulation. [S.I.], v. 99, n. 6, p. 779-785, 1999.

GUIMARÃES, N. G. et. al. Adesão a um programa de aconselhamento nutricional para adultos com excesso de peso e comorbidades. Rev Nutr. Campinas, v.23, n.3, p. 323-333, maio/jun. 2010.

IBGE. Pesquisa Nacional de Saúde 2013: Acesso e utilização dos serviços de saúde, acidentes e violências - Brasil, grandes regiões e unidades da federação. Rio de Janeiro: IBGE, 2015. Disponível em: <ftp://ftp.ibge.gov.br/PNS/2013/pns2013.pdf>. Acesso em: nov. 2018

JELLIFE, D B. Evaluacion del estado de nutricion de la comunidad: serie de monografias, n.53, Ginebra: [s.n.], 1968. 
MONTEIRO, C. A. et al. Diet quality, and health using the NOVA classification system. Roma: FAO, 2019.

OPAS - Organización Panamericana De La Salud. División de Promoción y Protección de la Salud. Encuesta Multicéntrica - Salud Bienestar y Envejecimiento (SABE) en América Latina y el Caribe - Informe Preliminar. Washington: [s.n.], mai. 2001.

OPAS - Organização Pan-Americana de Saúde. Doenças Cardiovasculares. Brasília: OPAS, [ca.2017]. Disponível em: <https://www.paho.org/pt/topicos/doencascardiovasculares>. Acesso em: nov. 2018

SANTOS, R. P. et al. Aconselhamento sobre Alimentação e Atividade Física: Prática e Adesão de Usuários da Atenção Primária. Rev Gaúcha Enf. [S.I.], v. 33, n. 4, 2012. p. 1421

SBC - Sociedade Brasileira de Cardiologia. Atualização Da Diretriz Brasileira De Dislipidemias E Prevenção Da Aterosclerose - 2017. Arq Bras de Card. [S.I.], v. 109, n. 1, 2017.

SBC - Sociedade Brasileira de Cardiologia.. I Diretriz Brasileira de Diagnóstico e Tratamento da Síndrome Metabólica. Arq Bras de Card. [S.I.], v. 84, supl. I., 2005.

SBC - Sociedade Brasileira de Cardiologia. VI Diretrizes Brasileiras de Hipertensão. Arq Bras Card. [S.I.], v. 95, n. 1, p. 1-51, 2010.

SBD - Sociedade Brasileira de Diabetes. OLIVEIRA. José E. P.; MOTENEGRO JUNIOR, R. M.; VENCIO, S. (Org.). Diretrizes Sociedade Brasileira de Diabetes 2017-2018. São Paulo: Editora Clannad, 2018. 383 p.

SBD - Sociedade Brasileira de Diabetes. José E. P.; VENCIO, S. (Org.). Diretrizes da Sociedade Brasileira de Diabetes 2013-2014. São Paulo: AC Farmacêutica, 2014. 382 p.

SPOSITO, Andrei C. et al. IV Diretriz Brasileira sobre Dislipidemias e Prevenção da Aterosclerose. Arq Bras Card. [S.I.], v. 88, n.2, supl. 1, p. 2-19, ago. 2007.

STEEMBURGO, T. et al. Fatores dietéticos e síndrome metabólica. Arq Bras Endocrinol Metab. São Paulo, v. 51, n. 9., p. 1425-1433, 2007.

TEREZA, J. et al. Development of a dietary index based on the Brazilian Cardioprotective Nutritional Program (BALANCE). Nutrition Journal. [S.I.: s.n.], 2018. p. 1-9. Disponível em: <https://doi.org/10.1186/s12937-018-0359-5>. Acesso em: dez. 2018

WEBER, B. et al. Effects of Brazilian Cardioprotective Diet Program on risk factors in patients with coronary heart disease: a Brazilian Cardioprotective Diet randomized pilot trial.

Clinics. [S.I.], v. 67, n. 12, p. 1407-1414, 2012. Disponível em: <http://clinics.org.br/article.php?id=913>. Acesso em: nov. 2018.

WEBER, B. et al. Implementation of a Brazilian Cardioprotective Nutritional (BALANCE ) Program for improvement on quality of diet and secondary prevention of cardiovascular events: A randomized, multicenter trial. American Heart Journal. [S.I.], v. 215, n.0, 2019.

WHO. Physical status: the use and interpretation of anthropometry. Report of a WHO Expert Committee. World Health Organization technical report series. National Library of Medicine. [S.I.: s.n.]: 1995. Disponível em: <https://pubmed.ncbi.nlm.nih.gov/8594834/>. Acesso em: dez. 2018. 